\title{
ONE MUST BREAK SYMMETRY IN ORDER TO KEEP ASSOCIATIVITY
}

\author{
HIDEKI OMORI \\ Department of Mathematics, Science University of Tokyo \\ 2641 Yamazaki, Noda-shi, Chiba-ken, 278-8510 Japan \\ E-mail: omori@ma.noda.sut.ac.jp
}

\begin{abstract}
Several anomalous facts about *-exponential functions of quadratic forms are discussed.
\end{abstract}

1. Introduction. It is widely accepted that the most fundamental notion in mathematics which deals with continuum is the calculus, where the calculus means the ordinary undergraduate calculus.

Let $u$ be the multiplication operator $x$. by the coordinate function $x$ on $\mathbf{R}$ acting on the space of all $\mathbf{C}$-valued $C^{\infty}$ functions on $\mathbf{R}$, and let $v$ be the operator $i \hbar \partial_{x}$. The operators $u$ and $v$ generate an algebra $W_{\hbar}$ over $\mathbf{C}$, called the Weyl algebra.

The Weyl algebra is an associative algebra generated by $u, v$ with the fundamental relation $[u, v]=-i \hbar$.

Note that we can change generators. For every $A \in S L(2, \mathbf{C})$, let

$$
\left[\begin{array}{l}
u^{\prime} \\
v^{\prime}
\end{array}\right]=A\left[\begin{array}{l}
u \\
v
\end{array}\right], \quad A \in S L(2, \mathbf{C}) .
$$

Then it is obvious that $\left[u^{\prime}, v^{\prime}\right]=-\hbar i$, and hence $u^{\prime}, v^{\prime}$ may also be viewed as generators. $u, v$, or $u^{\prime}, v^{\prime}$ will be called a canonical conjugate pair.

In this note, we point out several anomalous features of topological completions of the Weyl algebra under several topologies. For instance, $u$ has both a right inverse and a left inverse, and $u v$ has two different inverses.

The calculations for these facts are not so simple and not straightforward. The detailed systematic calculation will be published elsewhere.

2000 Mathematics Subject Classification: Primary 53D55, 53D10; Secondary 46L65.

Received 27 October 2000; revised 13 February 2001.

The paper is in final form and no version of it will be published elsewhere. 
2. Completion of free tensor algebras. The most universal associative algebra is the free tensor algebra. Taking the completion of the free tensor algebra under a suitable topology, and making the quotient by the closure of the relation ideal, we can obtain a complete topological associative algebra. This looks like the most universal way of obtaining complete topological algebras. However, this is not correct, as we show in this section.

Let $\mathcal{T}$ be the free tensor algebra generated by $X_{1}, X_{2}$. We introduce a topology into $\mathcal{T}$ so that $\mathcal{T}$ becomes a topological algebra.

An element of $\mathcal{T}$ is written in the form

$$
T=\sum c_{\alpha} X_{\alpha} \text { (finite sum), } \quad X_{\alpha}=X_{\alpha_{1}} \otimes X_{\alpha_{2}} \otimes \cdots \otimes X_{\alpha_{N}} \text { where } \alpha_{i} \in\{1,2\},
$$

$\alpha=\left(\alpha_{1}, \alpha_{2}, \cdots, \alpha_{N}\right) . X_{\alpha}$ is a word, and let $|\alpha|$ be the length $N$ of the word $X_{\alpha}$.

Define a system of semi-norms \|\|$_{\tau, s}(\tau, s>0)$ by

$$
\|T\|_{\tau, s}=\sum\left|c_{\alpha}\right||\alpha|^{\tau|\alpha|} s^{|\alpha|}
$$

For a fixed $\tau$, let $\mathcal{T}_{\tau}$ be the completion of $\mathcal{T}$ with respect to the topology induced by the system of semi-norms $\left\{\|\cdot\|_{\tau, s}\right\}_{s>0}$. Then one easily sees that

$$
\mathcal{T}_{\tau}=\left\{T=\sum_{|\alpha| \geq 0} c_{\alpha} X_{\alpha} \text { (formal); }\|T\|_{\tau, s}<\infty \text { for any } s>0\right\} .
$$

Lemma 1. For any $\tau \geq 0, \mathcal{T}_{\tau}$ becomes a Fréchet algebra satisfying

$$
\left\|T_{1} \otimes T_{2}\right\|_{\tau, s} \leq\left\|T_{1}\right\|_{\tau, e s}\left\|T_{2}\right\|_{\tau, e s} .
$$

To consider the topological completion of the Weyl algebra $W_{\hbar}$, we consider the twosided ideal $\mathcal{I}_{\hbar}$ generated by $X_{1} \otimes X_{2}-X_{2} \otimes X_{1}+\hbar i$, where $\hbar$ is a positive parameter. Let $\mathcal{I}_{\hbar, \tau}$ be the closure of $\mathcal{I}_{\hbar}$ in $\mathcal{T}_{\tau}$.

THEOREM 1. If $\tau \geq \frac{1}{2}$, then the quotient algebra $\mathcal{T}_{\tau} / \mathcal{I}_{\hbar, \tau}$ is a complete associative algebra containing the Weyl algebra $W_{\hbar}$ as a dense subalgebra.

However, if $\tau<\frac{1}{2}$, then the quotient algebra collapses to the trivial one, i.e. $1 \in \mathcal{I}_{\hbar, \tau}$, hence $\mathcal{T}_{\tau} / \mathcal{I}_{\hbar, \tau}=\{0\}$.

The proof of the first half is rather straightforward, but that of the second half is not. The proof deeply depends on some facts observed in the next section. Since quotient algebras are, by definition, associative, these cannot exhibit the anomalous phenomena mentioned in the introduction.

3. Extension of the product formula. There is another way of taking the topological completion of the Weyl algebra $W_{\hbar}$.

In general, there are so called ordering problems in the way of treating noncommutative algebras such as the Weyl algebra. The ordering problem may be viewed as the problem of expression of elements in a unique way. In the Weyl algebra, three kind of orderings: the normal ordering, the anti-normal ordering, and the Weyl ordering are mainly used. In the normal ordering we write elements in the form $\sum a_{m, n} u^{m} * v^{n}$ by arranging $u$ to the left hand side in each term. The anti-normal ordering is $\sum a_{m, n} v^{m} * u^{n}$. The Weyl ordering is when we write $\sum a_{m, n} u^{m} \circ v^{n}$ using the symmetric product $\circ$ defined by $u \circ v=\frac{1}{2}(u * v+v * u)$ 
etc. (See $[\mathrm{OMY}]$ for the details of symmetric product, but the reader need not care about the precise definition. This can be easily recovered from the inverse product formula given below.)

Through such an ordering, one can linearly identify the algebra with the space of all polynomials. In other words, through such an ordering, one can view the Weyl algebra as a non-commutative associative product structure defined on the space $\mathbf{C}[u, v]$ of all polynomials. The product formulas are given respectively as follows:

- In the normal ordering, the product $*$ of the Weyl algebra is given by the $\Psi$ DOproduct formula as follows:

$$
f(u, v) * g(u, v)=f \exp \left\{\hbar i\left(\overleftarrow{\partial_{v}} \circ \overrightarrow{\partial_{u}}\right)\right\} g=\sum_{n} \frac{(i \hbar)^{n}}{n !} \partial_{v}^{n} f \circ \partial_{u}^{n} g .
$$

- In the anti-normal ordering, the product $*$ of the Weyl algebra is given by the $\bar{\Psi} \mathrm{DO}-$ product formula as follows:

$$
f(u, v) * g(u, v)=f \exp \left\{-\hbar i\left(\overleftarrow{\partial_{u}} \bullet \overrightarrow{\partial_{v}}\right)\right\} g=\sum_{n} \frac{(-i \hbar)^{n}}{n !} \partial_{u}^{n} f \cdot \partial_{v}^{n} g
$$

- In the Weyl ordering, the product $*$ of the Weyl algebra is given by the Moyal product formula as follows:

$$
f(u, v) * g(u, v)=f \exp \frac{\hbar i}{2}\left\{\overleftarrow{\partial_{v}} \dot{\wedge} \overrightarrow{\partial_{u}}\right\} g,
$$

where $\overleftarrow{\partial_{v}} \dot{\wedge} \overrightarrow{\partial_{u}}=\overleftarrow{\partial_{v}} \cdot \overrightarrow{\partial_{u}}-\overleftarrow{\partial_{u}} \cdot \overrightarrow{\partial_{v}}$. Every product formula yields $u * v-v * u=-\hbar i$ and hence defines the Weyl algebra. Here, the commutative products $\circ, \bullet, \cdot$ play only a supplementary role to express elements in a unique way. We distinguish these to indicate which ordering is used.

To justify these, we remark the following: Define derivations on the Weyl algebra by $\partial_{u}=\frac{1}{\hbar i} \operatorname{ad}(v), \partial_{v}=-\frac{1}{\hbar i} \operatorname{ad}(u)$. Then the inverse product formulas

$$
\begin{gathered}
f(u, v) \circ g(u, v)=f \exp \left\{-\hbar i\left(\overleftarrow{\partial_{v}} * \overrightarrow{\partial_{u}}\right)\right\} g, \quad f(u, v) \bullet g(u, v)=f \exp \left\{\hbar i\left(\overleftarrow{\partial_{u}} * \overrightarrow{\partial_{v}}\right)\right\} g \\
f(u, v) \cdot g(u, v)=f \exp \frac{\hbar i}{2}\left\{\overleftarrow{\partial_{u}} * \overrightarrow{\partial_{v}}-\overleftarrow{\partial_{v}} * \overrightarrow{\partial_{u}}\right\} g,
\end{gathered}
$$

called the inverse $\Psi$ DO-, inverse $\bar{\Psi}$ DO-, and inverse Moyal product formula respectively, give associative commutative algebras, isomorphic to the ordinary polynomial algebra. Hence, these commutative products are defined by using the $*$-product only.

The replacement (pull-back) $A^{*}$ of $u, v$ by $u^{\prime}, v^{\prime}$ gives an algebra isomorphism of $W_{\hbar}$. Thus, we may consider the ordering problem by using $u^{\prime}, v^{\prime}$ instead of $u, v$.

The following is the most useful property of the Moyal product formula:

Proposition 2. For every $A \in S L(2, \mathbf{C})$, let $\Phi^{*}$ be the replacement (pull-back) of $u, v$ into $u^{\prime}, v^{\prime}$ by the combination of the linear transformation with matrix $A$ and a parallel displacement:

$$
\left[\begin{array}{l}
u^{\prime} \\
v^{\prime}
\end{array}\right]=A\left[\begin{array}{l}
u \\
v
\end{array}\right]+\left[\begin{array}{l}
\alpha \\
\beta
\end{array}\right], \quad A \in S L(2, \mathbf{C}), \quad(\alpha, \beta) \in \mathbf{C}^{2} .
$$

Then, $\Phi^{*}$ is an isomorphism for both *-and--products. 
Note that other expressions do not have this property. It is easily seen that if $a b \neq 0$, then

$$
(a u+b v)_{*}^{m}=(a u+b v)_{.}^{m}, \text { but }(a u+b v)_{*}^{m} \neq(a u+b v)_{\circ}^{m} .
$$

In spite of this, we have the following formula for the normal ordering:

$$
f(u) * g(u, v)=f(u) \circ g(u, v), \quad f(u, v) * g(v)=f(u, v) \circ g(v) .
$$

REMARK 1. Several careful computations are required to fix the intertwiners between different orderings, or between normal orderings using different canonical conjugate pairs. Precise formulas will be given in another paper.

Since all product formulas are given by concrete forms, these extend to the following: Let $\operatorname{Hol}\left(\mathbf{C}^{2}\right)$ be the space of all entire functions on $\mathbf{C}^{2}$ with the compact open topology.

- $f * g$ is defined if one of $f, g$ is a polynomial.

- For every polynomial $p=p(u, v)$, the left (resp. right) multiplication $p *$ (resp. $* p$ ) is a continuous linear mapping of $\operatorname{Hol}\left(\mathbf{C}^{2}\right)$ into itself with respect to the compact open topology.

- By the polynomial approximation theorem, the associativity $f *(g * h)=(f * g) * h$ holds if two of $f, g, h$ are polynomials.

We call such a system a twosided $(\mathbf{C}[u, v] ; *)$-module.

REMARK 2. If one computes everything as formal power series of $\hbar$, then the product $f * g$ is well defined and gives an associative algebra. Hence, if $f * g$ is defined as a real analytic function in $\hbar \in[0, T), T>0$, then the associativity holds.

Starting from a twosided $(\mathbf{C}[u, v] ; *)$-module, the $*$-product extends to a wider class of functions. For every positive $p>0$, set

$$
\mathcal{E}_{p}\left(\mathbf{C}^{2}\right)=\left\{f \in \operatorname{Hol}\left(\mathbf{C}^{2}\right) ;\|f\|_{p, s}=\sup |f| e^{-s|\xi|^{p}}<\infty, \forall s>0\right\}
$$

where $|\xi|=\left(|u|^{2}+|v|^{2}\right)^{1 / 2}$. The family $\left\{\|\|_{p, s}\right\}_{s>0}$ induces a topology on $\mathcal{E}_{p}\left(\mathbf{C}^{2}\right)$ and $\left(\mathcal{E}_{p}\left(\mathbf{C}^{2}\right), \cdot\right)$ is an associative commutative Fréchet algebra, where the dot $\cdot$ is the ordinary multiplication for functions in $\mathcal{E}_{p}\left(\mathbf{C}^{2}\right)$. Thus, - may be replaced by $\circ$ or $\bullet$ to indicate the ordering. It is easily seen that for $0<p<p^{\prime}$, we have a continuous embedding

$$
\mathcal{E}_{p}\left(\mathbf{C}^{2}\right) \subset \mathcal{E}_{p^{\prime}}\left(\mathbf{C}^{2}\right)
$$

as a commutative Fréchet algebra (cf. [GS]), and that $\mathcal{E}_{p}\left(\mathbf{C}^{2}\right)$ is $S L(2, \mathbf{C})$-invariant under the natural linear action (1).

It is obvious that every polynomial is contained in $\mathcal{E}_{p}\left(\mathbf{C}^{2}\right)$ and $\mathbf{C}[u, v]$ is dense in $\mathcal{E}_{p}\left(\mathbf{C}^{2}\right)$ for any $p>0$ in the Fréchet topology defined on it.

We remark that every exponential function $e^{\alpha u+\beta v}$ is contained in $\mathcal{E}_{p}\left(\mathbf{C}^{2}\right)$ for any $p>1$, but not in $\mathcal{E}_{1}\left(\mathbf{C}^{2}\right)$, and functions such as $e^{a u^{2}+b v^{2}+2 c u v}$ are contained in $\mathcal{E}_{p}\left(\mathbf{C}^{2}\right)$ for any $p>2$, but not in $\mathcal{E}_{2}\left(\mathbf{C}^{2}\right)$. Functions such as $\sum \frac{1}{(n !)^{1 / p}} u^{n}$ are contained in $\mathcal{E}_{q}\left(\mathbf{C}^{2}\right)$ for any $q>p$, but not in $\mathcal{E}_{p}\left(\mathbf{C}^{2}\right)$.

TheOREm 3. For $0<p \leq 2, \mathcal{E}_{p}\left(\mathbf{C}^{2}\right)$ is closed under the product $*$, and $\left(\mathcal{E}_{p}\left(\mathbf{C}^{2}\right) ; *\right)$ is isomorphic to $\mathcal{T}_{1 / p} / \mathcal{I}_{\hbar, 1 / p}$.

For $p>2$, we have the following by choosing $p^{\prime}$ such that $1 / p+1 / p^{\prime} \geq 1$ : 
THEOREM 4. Each product formula stated above gives continuous bilinear maps

$$
\mathcal{E}_{p}\left(\mathbf{C}^{2}\right) \times \mathcal{E}_{p^{\prime}}\left(\mathbf{C}^{2}\right) \rightarrow \mathcal{E}_{p}\left(\mathbf{C}^{2}\right), \quad \mathcal{E}_{p^{\prime}}\left(\mathbf{C}^{2}\right) \times \mathcal{E}_{p}\left(\mathbf{C}^{2}\right) \rightarrow \mathcal{E}_{p}\left(\mathbf{C}^{2}\right)
$$

4. Associativity breaks down in $\mathcal{E}_{2+}\left(\mathbf{C}^{2}\right)$. Set $\mathcal{E}_{2+}\left(\mathbf{C}^{2}\right)=\bigcap_{p>2} \mathcal{E}_{p}\left(\mathbf{C}^{2}\right)$. Reflecting the fact stated in Theorem $1, \mathcal{E}_{2+}\left(\mathbf{C}^{2}\right)$ is not closed under the product $*$. Moreover, several strange phenomena occur in the twosided $\mathcal{E}_{p^{\prime}}\left(\mathbf{C}^{2}\right)$-module $\mathcal{E}_{2+}\left(\mathbf{C}^{2}\right)$ for $p^{\prime}<2$.

A direct calculation using the Moyal product formula shows that the coordinate function $v$ has a right inverse $v^{\circ}=\frac{1}{v}\left(1-e^{\frac{2 i}{\hbar} u v}\right)$, and a left inverse $v^{\bullet}=\frac{1}{v}\left(1-e^{-\frac{2 i}{\hbar} u v}\right)$ as the complex conjugate of the right inverse given above. Here the complex conjugate means the anti-homomorphism generated by $\bar{u}=u, \bar{v}=v, \bar{i}=-i$ etc. These elements are in $\mathcal{E}_{2+}\left(\mathbf{C}^{2}\right)$.

If the associativity holds, then these should be the same genuine inverse. Hence we must set

$$
\frac{\hbar}{2 i}\left(v^{\circ}-v^{\bullet}\right)=\frac{1}{v} \sin \frac{2}{\hbar} u v=0 .
$$

Since this is impossible, we loose also the associativity in $\mathcal{E}_{2+}\left(\mathbf{C}^{2}\right)$.

On the other hand, we easily see by the Moyal product formula that

$$
v * e^{\frac{2 i}{\hbar} u v}=0=e^{\frac{2 i}{\hbar} u v} * u, \quad u * e^{-\frac{2 i}{\hbar} u v}=0=e^{-\frac{2 i}{\hbar} u v} * v .
$$

We call $2 e^{\frac{2 i}{\hbar} u v}$ a vacuum and $2 e^{-\frac{2 i}{\hbar} u v}$ a bar-vacuum and denote these by $\varpi_{00}$ and $\bar{\varpi}_{00}$ respectively, but a bar-vacuum $\bar{\varpi}_{00}$ is a vacuum with respect to the canonical conjugate pair $(-v, u)$.

Although the associativity breaks down in general, we can show that

$$
\left(u^{p} * \varpi_{0,0} * v^{q}\right) *\left(u^{r} * \varpi_{0,0} * v^{s}\right)=\delta_{q, r} q !(\hbar i)^{q} * u^{p} * \varpi_{0,0} * v^{s} .
$$

Hence $E_{p, q}=\frac{1}{\sqrt{(\hbar i)^{p+q} p ! q !}} u^{p} * \varpi_{0,0} * v^{q}, p, q \in \mathbf{N}$, form matrix elements. Hence

$$
\left\{E_{p, q} ; p, q \in \mathbf{N}\right\}
$$

spans an associative algebra contained in $\left(\mathcal{E}_{2+}\left(\mathbf{C}^{2}\right) ; *\right)$, and this is isomorphic to the matrix algebra $M(\infty)=\operatorname{injlim} M(k)$.

Note the following:

Proposition 5. By Zorn's lemma, we can consider a maximal associative algebra in the space $\left(\mathcal{E}_{2+}\left(\mathbf{C}^{2}\right) ; *\right)$.

The space $\left(\mathcal{E}_{2+}\left(\mathbf{C}^{2}\right) ; *\right)$ contains many maximal associative algebra with respect to the $*$-product, but there is no standard manner to choose such an associative algebra. The above observation shows that a half-inverse cannot exist in a non-commutative associative algebra with an involutive anti-automorphism such as complex conjugation.

5. Star-exponential functions of quadratic forms. These strange phenomena relate to $*$-exponential functions of quadratic forms. Note that the exponential function $e_{*}^{t\left(a u^{2}+b v^{2}+2 c u v\right)}$ is defined by the following evolution equation: Set $e_{*}^{t\left(a u^{2}+b v^{2}+2 c u v\right)}=$ $F(t, u, v)$,

$$
\frac{\partial}{\partial t} F(t, u, v)=\left(a u^{2}+b v^{2}+2 c u v\right) * F(t, u, v), \quad F(0, u, v)=1
$$


The right hand side is computed by the Moyal product formula as follows:

$$
\begin{aligned}
\left(a u^{2}+b v^{2}+2 c u v\right) & * F(t, u, v)=\left(a u^{2}+b v^{2}+2 c u v\right) F \\
+ & \hbar i\left\{(b v+c u) \partial_{u} F-(a u+c v) \partial_{v} F\right\}-\frac{\hbar^{2}}{4}\left\{b \partial_{u}^{2} F-2 c \partial_{v} \partial_{u} F+a \partial_{v}^{2} F\right\}
\end{aligned}
$$

This is a partial differential equation. If $a b-c^{2}>0$, then this is the heat equation and the existence of solutions is not ensured in general. This implies that the mapping $f(u, v) \rightarrow e_{*}^{\frac{t}{\hbar}\left(a u^{2}+b v^{2}+2 c u v\right)} * f(u, v)$ is not always defined. However, we see that a real analytic solution in $t$ is unique, if it exists.

Hence we assume that $e_{*}^{t\left(a u^{2}+b v^{2}+2 c u v\right)}$ is a function of $a u^{2}+b v^{2}+2 c u v$; that is,

$$
e_{*}^{t\left(a u^{2}+b v^{2}+2 c u v\right)}=f_{t}\left(a u^{2}+b v^{2}+2 c u v\right) .
$$

Then, setting $x=a u^{2}+b v^{2}+2 c u v$, we have

$$
\frac{d}{d t} f_{t}(x)=x f_{t}(x)-\hbar^{2}\left(a b-c^{2}\right)\left(f_{t}^{\prime}(x)+x f_{t}^{\prime \prime}(x)\right) .
$$

The right hand side is the Bessel operator.

However, there is another method to treat this differential equation, called the method of Lie groups. We assume that

$$
e_{*}^{t\left(a u^{2}+b v^{2}+2 c u v\right)}=s(t) e^{\left.a(t) u^{2}+b(t) v^{2}+2 c(t) u v\right)},
$$

then we only have to solve a system of ordinary differential equations.

LEMma 2. The solution of (6) with the initial function 1 is given by

$$
\frac{1}{\cosh \left(\hbar \sqrt{a b-c^{2}} t\right)} \exp \left\{\frac{x}{\hbar \sqrt{a b-c^{2}}} \tanh \left(\hbar \sqrt{a b-c^{2}} t\right)\right\} \text {. }
$$

If $a b-c^{2}=0$, then we set

$$
\frac{1}{\hbar \sqrt{a b-c^{2}}} \tanh \left(\hbar \sqrt{a b-c^{2}} t\right)=t .
$$

Note that if $c^{2}-a b=1$, then $e_{*}^{t\left(a u^{2}+b v^{2}+2 c u v\right)}$ is singular at $t= \pm \frac{\pi}{2 \hbar}$. This expression appears also in $[\mathrm{MS}]$ in a different context. The uniqueness of the real analytic solution gives the exponential law if both sides are defined. In particular,

$$
e^{\frac{s}{2 \hbar} u v} * e^{\frac{t}{2 \hbar} u v}=\frac{1}{1-s t} e^{\frac{s+t}{1-s t} u v} .
$$

The formula of $e_{*}^{t\left(a u^{2}+b v^{2}+2 c u \circ v\right)}$ under the normal ordering with respect to the canonical conjugate pair $u, v$ is a little complicated to write down. We give here some of them:

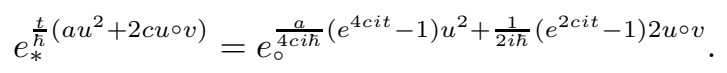

In particular, we see that $e_{*}^{\frac{\pi}{\hbar}\left(a u^{2}+u \circ v\right)}$ does not depend on $a$ :

$$
e_{*}^{\frac{\pi}{2 \hbar}\left(a u^{2}+2 u \circ v\right)}=e_{\circ}^{-\frac{1}{\hbar i} 2 u \circ v}=e_{*}^{\frac{\pi}{\hbar} u * v} .
$$

By the exponential law, we have in particular the following product formula:

$$
e_{\circ}^{\frac{1}{\hbar i} s u \circ v} * e_{\circ}^{\frac{1}{\hbar i} t u \circ v}=e_{\circ}^{\frac{1}{\hbar i}(s+t+s t) u \circ v} .
$$


Similarly, we have

$$
e_{*}^{\frac{t}{\hbar}\left(b v^{2}+2 c u \circ v\right)}=e_{\circ}^{\frac{b}{4 c i \hbar}\left(e^{4 c i t}-1\right) v^{2}+\frac{1}{2 \hbar i}\left(e^{2 c i t}-1\right) 2 u \circ v} .
$$

In particular, $e_{*}^{\frac{\pi}{\hbar}\left(b v^{2}+2 u \circ v\right)}$ does not depend on $b$.

Note that if $c^{2}-a b=1$, then

$$
a u^{2}+b v^{2}+2 c u v=(\alpha u+\beta v)(\gamma u+\delta v), \quad \alpha \delta-\beta \gamma=2 .
$$

Note also that $[\alpha u+\beta v, \gamma u+\delta v]=-2 \hbar i$. Hence, $e_{*}^{t\left(a u^{2}+b v^{2}+2 c u v\right)}$ is treated in the normal ordering with respect to the canonical conjugate pair

$$
u^{\prime}=\frac{1}{\sqrt{2}}(\alpha u+\beta v), \quad v^{\prime}=\frac{1}{\sqrt{2}}(\gamma u+\delta v) .
$$

By (7), (8) we see the following:

THEOREM 6. Although $e_{*}^{t\left(a u^{2}+b v^{2}+2 c u v\right)}$ is singular at $t= \pm \frac{\pi}{2 \hbar}$ in the Weyl ordering, for every $a, b, c$ such that $c^{2}-a b=1$, it is not singular in a suitably chosen normal ordering.

Consider the set

$$
\varepsilon_{00}=\left\{e_{*}^{\frac{\pi}{2 \hbar}\left(a u^{2}+b v^{2}+2 c u v\right)} ; c^{2}-a b=1\right\} .
$$

Though the set $\varepsilon_{00}$ looks like forming a 2-dimensional subset, the particular cases of (7) and (8) show that it is in fact a degenerate set having the following properties:

THEOREM 7. $\varepsilon_{00}$ is formed by a single point with the following property: $\varepsilon_{00} * \varepsilon_{00}=-1$, $u * \varepsilon_{00}+\varepsilon_{00} * u=0, v * \varepsilon_{00}+\varepsilon_{00} * v=0$.

The normal ordering expression of $\varepsilon_{00}$ with respect to a canonical conjugate pair $\left(u^{\prime}, v^{\prime}\right)=(\alpha u+\beta v, \gamma u+\delta v)$ is $\varepsilon_{00}=i e_{\circ}^{\frac{2 i}{\hbar} u^{\prime} \circ v^{\prime}}$ where $\circ$ is the ordinary commutative product identified with the normal ordering with respect to $\left(u^{\prime}, v^{\prime}\right)$.

$\varepsilon_{00}$ will be called the polar element. The proof of this fact is given by computing $\varepsilon_{00}$ in normal orderings with respect to various canonical conjugate pairs.

Quadratic forms $\left\{a u^{2}+b v^{2}+2 c u v\right\}$ are closed under the commutator bracket, and form a Lie algebra $\operatorname{sl}(2 ; \mathbf{C})$.

TheOREM 8. $e_{*}^{t\left(a u^{2}+b v^{2}+2 c u v\right)}$ is contained in $\mathcal{E}_{2+}\left(\mathbf{C}^{2}\right) \cup \varepsilon_{00} * \mathcal{E}_{2+}\left(\mathbf{C}^{2}\right)$ for every $a, b, c \in$ C. If one identifies the \pm 1 multiplication factors, then $e_{*}^{t\left(a u^{2}+b v^{2}+2 c u v\right)}$ generates a Lie group which is isomorphic to $S L(2 ; \mathbf{C})$.

6. *-delta functions, $*$-Heaviside functions. Recall that $e_{*}^{\frac{t}{\hbar} u \cdot v}$ is defined by the equation

$$
\frac{d}{d t} f_{t}(u, v)=\frac{1}{\hbar}(u \cdot v) * f_{t}(u, v), \quad f_{0}(u, v)=1 .
$$

The solution is given by $\frac{1}{\cosh \frac{t}{2}} e^{\frac{i}{\hbar}\left(\tanh \frac{t}{2}\right) 2 u \cdot v}$ in the Weyl ordering. Hence,

$$
\int_{0}^{\infty} \frac{1}{\cosh \frac{t}{2}} e^{\frac{i}{\hbar}\left(\tanh \frac{t}{2}\right) 2 u \cdot v} d t, \quad \int_{-\infty}^{0} \frac{1}{\cosh \frac{t}{2}} e^{\frac{i}{\hbar}\left(\tanh \frac{t}{2}\right) 2 u \cdot v} d t
$$


exist in the space $\mathcal{E}_{2+}\left(\mathbf{C}^{2}\right)$. It follows that $u \cdot v$ has two different inverses as follows:

$$
(u \cdot v)_{+i 0}^{-1}=-i \int_{0}^{\infty} e_{*}^{\frac{i t}{\hbar} u \cdot v} d t, \quad(u \cdot v)_{-i 0}^{-1}=i \int_{-\infty}^{0} e_{*}^{\frac{i t}{\hbar} u \cdot v} d t .
$$

The difference is given by using the Bessel function $J_{0}$ :

$$
(u \cdot v)_{+i 0}^{-1}-(u \cdot v)_{-i 0}^{-1}=-i \int_{-\infty}^{\infty} e_{*}^{\frac{i t}{\hbar} u \cdot v} d t=\frac{\pi}{2 i} J_{0}\left(\frac{2}{\pi} u \cdot v\right)
$$

which may be viewed as the $*$-delta function $-i \delta_{*}(u \cdot v)$. Hence the associativity must break down, and

$$
(u \cdot v) * \delta_{*}(u \cdot v)=\delta_{*}(u \cdot v) *(u \cdot v)=0 .
$$

Thus, it is impossible to treat $(u \cdot v)_{+i 0}^{-1}$ and $(u \cdot v)_{-i 0}^{-1}$ in the same associative algebra. Indeed, we see that $(u \cdot v)_{+i 0}^{-1} *(u \cdot v)_{-i 0}^{-1}$ diverges.

Note that if $z$ is the complex coordinate function on $\mathbf{C}$, then

$$
(u \cdot v-z)_{+i 0}^{-1}=-i \int_{0}^{\infty} e_{*}^{\frac{i t}{\hbar}(u \cdot v-z)} d t, \quad(u \cdot v-z)_{-i 0}^{-1}=i \int_{-\infty}^{0} e_{*}^{\frac{i t}{\hbar}(u \cdot v-z)} d t
$$

are holomorphic on the domain $\{z ; \operatorname{Im} z>-\hbar / 2\},\{z ; \operatorname{Im} z<\hbar / 2\}$ respectively. Thus, the $*$-delta function $\delta_{*}(u \cdot v)$ is expressed as the difference of the boundary values of two holomorphic functions (cf. $[\mathrm{M}])$.

Since $u * v=u \cdot v-\frac{\hbar i}{2}$ and $e_{*}^{i t u * v}=e_{*}^{i t u \cdot v} e^{\frac{\hbar t}{2}}$ is proved by the uniqueness of a real analytic solution, we see that

$$
\varpi_{00}=\lim _{t \rightarrow \infty} e_{*}^{i t u * v} .
$$

Hence this element is explained as an equilibrium state in [BL].

By (4), we see

$$
\left(u v-\frac{\hbar i}{2}\right) * e^{\frac{2 i}{\hbar} u v}=u * v * e^{\frac{2 i}{\hbar} u v}=0 .
$$

However, $u v-\frac{\hbar i}{2}$ has an inverse contained in $\mathcal{E}_{2+}\left(\mathbf{C}^{2}\right)$. Thus, the associativity fails again in $\mathcal{E}_{2+}\left(\mathbf{C}^{2}\right)$ :

$$
\left(\left(u v-\frac{\hbar i}{2}\right)^{-1} *\left(u v-\frac{\hbar i}{2}\right)\right) * e^{\frac{2 i}{\hbar} u v} \neq\left(u v-\frac{\hbar i}{2}\right)^{-1} *\left(\left(u v-\frac{\hbar i}{2}\right) * e^{\frac{2 i}{\hbar} u v}\right) .
$$

Thus, $u v-\frac{\hbar i}{2}$ and $\varpi_{00}$ cannot be elements of an associative algebra at the same time. But, this is the same as to say that $\varpi_{00}$ and $\bar{\varpi}_{00}$ cannot be elements of an associative algebra at the same time.

Define the $*$-Heaviside function by

$$
H_{*}(u v)=\frac{1}{2 \pi} \int_{-\infty}^{\infty} \frac{1}{c+i t} e_{*}^{i t u v} d t, \quad c>0 .
$$

Note that $H_{*}(u v) \in \mathcal{E}_{2+}\left(\mathbf{C}^{2}\right)$ and $H_{* \pm}(u v-z)=\frac{1}{2 \pi} \int_{0}^{\infty} \frac{1}{c \pm i t} e_{*}^{i t(u v-z)} d t$ are holomorphic functions respectively on the upper half-plane $H_{+}$and the lower half-plane $H_{-}$, and $H_{*}$ is viewed as the difference of boundary values, $H_{*+}-H_{*-}$.

By using the exponential law, it is not hard to see that

$$
\begin{gathered}
H_{*}(u v) * H_{*}(u v)=H_{*}(u v), \quad H_{*}(u v) * H_{*}(-u v)=0, \\
H_{*}(u v)+H_{*}(-u v)=1, \quad H_{*}^{\prime}(u v)=\delta_{*}(u v) .
\end{gathered}
$$


On the other hand, computing the adjoint action

$$
e_{*}^{t\left(u^{2}-v^{2}\right)} * f(u v) * e_{*}^{-t\left(u^{2}-v^{2}\right)}=\exp \left\{t \operatorname{ad}\left(u^{2}-v^{2}\right)\right\} f(u v)
$$

we obtain the exchange rule

$$
e_{*}^{\frac{\pi}{4}\left(u^{2}-v^{2}\right)} * f(u v)=f(-u v) * e_{*}^{\frac{\pi}{4}\left(u^{2}-v^{2}\right)} .
$$

Set

$$
\xi=e_{*}^{\frac{\pi}{4}\left(u^{2}-v^{2}\right)} * H_{*}(u v), \quad \eta=H_{*}(u v) * e_{*}^{-\frac{\pi}{4}\left(u^{2}-v^{2}\right)} .
$$

After checking the associativity via Remark 2, we have the formulas

$$
\xi^{2}=\eta^{2}=0, \quad \xi * \eta+\eta * \xi=1 .
$$

This gives that the $2 \times 2$-matrix algebra $M(2 ; \mathbf{C})$, and hence $S L(2 ; \mathbf{C})$ is contained in $\mathcal{E}_{2+}\left(\mathbf{C}^{2}\right)$. However, this is not the same group given in Theorem 8 .

7. Vacuum representations. In this section, we give the most popular way to choose an associative algebra contained in $\mathcal{E}_{2+}\left(\mathbf{C}^{2}\right)$.

We set $\varpi_{00}=2 e^{\frac{2 i}{\hbar} u v}$, and $\bar{\varpi}_{00}=2 e^{-\frac{2 i}{\hbar} u v}$. By the Moyal product formula, we see easily that

$$
v * \varpi_{00}=0=\varpi_{00} * u, \quad u * \bar{\varpi}_{00}=0=\bar{\varpi}_{00} * v .
$$

Thus, $\varpi_{00}$ plays the same role as the vacuum $|0\rangle$ such that $v|0\rangle=0$.

By a direct calculation using the Moyal product formula, we have for every entire function $f(u)$ of one variable that

$$
f(u) * \varpi_{00}=f(2 u) * \varpi_{00}, \quad \varpi_{00} * f(u)=f(0) .
$$

Let $\mathcal{E}_{2+}\left(\mathbf{C}_{u}\right)$ be the subspace consisting of all $f(u) \in \mathcal{E}_{2+}\left(\mathbf{C}^{2}\right)$. Hence, we have

Proposition 9. $\mathcal{E}_{2+}\left(\mathbf{C}_{u}\right) * \varpi_{00} \subset \mathcal{E}_{2+}\left(\mathbf{C}^{2}\right)$.

The point is the following fact, proved by polynomial approximation:

Proposition 10. For any entire function $h(u) \in \operatorname{Hol}(\mathbf{C})$ and any polynomial $p(u, v)$, the associativity

$$
p(u, v) *\left(h(u) * \varpi_{00}\right)=(p(u, v) * h(u)) * \varpi_{00}
$$

holds.

For any polynomial $p_{\circ}(u, v)$ in the normal ordering, the linear operator $L\left(p_{\circ}\right)$ defined by

$$
h(u) * \varpi_{00} \mapsto p_{\circ}(u, v) * h(u) * \varpi_{00}=p_{\circ}\left(u, \hbar i \partial_{u}\right) * h(u) * \varpi_{00}
$$

is a continuous linear operator of $\operatorname{Hol}(\mathbf{C}) * \varpi_{00}$ into itself with respect to the compact open topology.

Proposition 11. If $L\left(p_{\circ n}\right)$ converges weakly to a continuous linear operator $L$ on $\operatorname{Hol}(\mathbf{C}) * \varpi_{00}$, then there is $f_{\circ} \in \operatorname{Hol}(\mathbf{C})$ such that $\lim p_{\circ n}=f_{\circ}$ in the compact open topology. 
We define $L\left(f_{\circ}(u, v)\right)=\lim L\left(p_{\circ}(u, v)\right)$. This is justified by the fact that the $*-$ product $f_{\circ} *\left(h(u) * \varpi_{0,0}\right)$ is defined by

$$
f_{\circ}(u, v) *\left(h(u) * \varpi_{00}\right)=\lim p_{\circ n}(u, v) * h(u) * \varpi_{00} .
$$

Let $\mathcal{A}\left(\varpi_{00}\right)$ be the totality of $f_{\circ} \in \operatorname{Hol}\left(\mathbf{C}^{2}\right)$ such that there is a polynomial approximation $p_{\circ n}$ of $f$ such that $L\left(p_{\circ n}\right)$ weakly converges to a continuous linear operator $L$ from $\operatorname{Hol}(\mathbf{C}) * \varpi_{00}$ into itself.

The following is one of the most popular ways to choose an associative algebra:

THEOREM 12. Extending the product * by

$$
L\left(f_{\circ}\right) L\left(g_{\circ}\right)=L\left(f_{\circ} * g_{\circ}\right),
$$

$\mathcal{A}\left(\varpi_{00}\right)\left(\subset \operatorname{Hol}\left(\mathbf{C}^{2}\right)\right)$ forms an associative algebra whose product is an extension of the $*$ product defined on the space of polynomials.

For every compactly supported smooth function $\hat{f}(t)$ on $\mathbf{R}$, the Fourier transform

$$
f_{*}(u)=\frac{1}{\sqrt{2 \pi}} \int_{\mathbf{R}} \hat{f}(t) e_{*}^{i t u} d t
$$

gives an entire function. We have

$$
\varpi_{00} * f_{*}(u) * \varpi_{00}=\int_{\mathbf{R}} \hat{f}(t) d t .
$$

Hence, the notion of trace is naturally involved in our system. If

$$
\bar{g}_{*}(u)=\frac{1}{\sqrt{2 \pi}} \int_{\mathbf{R}} \overline{\hat{g}}(t) e_{*}^{i t u} d t
$$

then we define the inner product by

$$
\left\langle f_{*}, g_{*}\right\rangle=\varpi_{00} * f_{*}(u) * \bar{g}_{*}(u) * \varpi_{00} .
$$

Here is the list of vacuum representation of several elements:

$$
\begin{gathered}
u=x \cdot \quad v=i \hbar \partial_{x}, \quad v^{\circ}=\frac{1}{i \hbar} \int_{0}^{x} d x, \\
u^{\bullet}(f)(x)=\frac{1}{x}(f(x)-f(0)), \quad \varpi_{00}(f)(x)=f(0),
\end{gathered}
$$

and

$$
\varepsilon_{00}(f)(x)=i f(-x) .
$$

However, $\bar{\varpi}_{00}, u^{\circ}, v^{\bullet}, \delta_{*}, H_{*}$ etc. are not expressed in the vacuum representation.

\section{References}

[BL] H. Basart and A. Lichnerowicz, Conformal symplectic geometry, deformations, rigidity and geometrical KMS conditions, Lett. Math. Phys. 10 (1985), 167-177.

[GS] I. M. Gel'fand and G. E. Shilov, Generalized Functions, 2, Academic Press, 1968.

[M] M. Morimoto, An Introduction to Sato's Hyperfunctions, AMS, 1993.

[MS] C. Moreno and J. A. P. da Silva, Star products, spectral analysis and hyperfunctions, in: Conférence Moshé Flato 1999, Quantizations, Deformations, and Symmetries, Vol. II, Kluwer, 2000, 211-214. 
[Om] H. Omori, Infinite Dimensional Lie Groups, AMS, 1997.

[OMY] H. Omori, Y. Maeda and A. Yoshioka, Weyl manifolds and deformation quantization, Adv. Math. 85 (1991), 224-255.

[O,el] H. Omori, Y. Maeda, N. Miyazaki and A. Yoshioka, Deformation quantization of Fréchet-Poisson algebras-Convergence of the Moyal product, in: Conférence Moshé Flato 1999, Quantizations, Deformations, and Symmetries, Vol. II, Kluwer, 2000, 233246. 